\title{
Possible quantum nematic phase in a colossal magnetoresistance material
}

\author{
G. Beaudin $\odot$, L. M. Fournier, and A. D. Bianchi $\odot$ \\ Département de Physique, Université de Montréal, Montréal, Canada* \\ M. Nicklas (루 \\ Max Planck Institute for Chemical Physics of Solids, Dresden, Germany \\ M. Kenzelmann \\ Laboratory for Neutron Scattering and Imaging, Paul Scherrer Institut, Villigen, Switzerland \\ M. Laver \\ Department of Physics, University of Warwick, Coventry, United Kingdom \\ W. Witczak-Krempa \\ Département de Physique, Université de Montréal, Montréal, Canada* \\ and Centre de Recherches Mathématiques, Université de Montréal, Montréal, Québec, Canada
}

(Received 17 September 2019; revised 14 December 2021; accepted 15 December 2021; published 3 January 2022)

\begin{abstract}
$\mathrm{EuB}_{6}$ has for a long time captured the attention of the physics community, as it shows a ferromagnetic phase transition leading to a insulator the metal transition together with colossal magnetoresistance (CMR). EuB 6 has a very low carrier density, which is known to drastically change the interaction between the localised Eu moments and the conduction electrons. One of early triumphs of the quantum theory in condensed matter was the presence of Fermi surface, which is intimately linked to the symmetry of the underlying crystal lattice. This symmetry can be probed by angle dependent magnetoresistance oscillations (AMRO) measurements. Here, we present $\mathrm{AMRO}$ measurements that show that in $\mathrm{EuB}_{6}$ this symmetry is broken, possibly indicating the presence of a quantum nematic phase. We identify the region in the temperature-magnetic field phase diagram where the magnetoresistance shows twofold oscillations instead of the expected fourfold pattern. Quantum nematic phases are analogous to classical liquid crystals. Like liquid crystals, which break the rotational symmetry of space, their quantum analogs break the point-group symmetry of the crystal due to strong electron-electron interactions, as in quantum Hall states, $\mathrm{Sr}_{3} \mathrm{Ru}_{2} \mathrm{O}_{7}$, and high-temperature superconductors. This is the same region where magnetic polarons were previously observed, suggesting that they drive the nematicity in $\mathrm{EuB}_{6}$. This is also the region of the phase diagram where $\mathrm{EuB}_{6}$ shows a colossal magnetoresistance (CMR). This novel interplay between magnetic and electronic properties could thus be harnessed for spintronic applications.
\end{abstract}

DOI: 10.1103/PhysRevB.105.035104

\section{INTRODUCTION}

One of the great successes of the quantum theory of solids was the finding that electrons in crystals largely behave as a quantum gas of free particles. It was soon understood that the interactions between electrons can change their behavior into that of a quantum liquid leading among other things to a modified mass of the quasiparticles. However, unlike in classical liquids, where a suspension of rod-like molecules can lead to anisotropic interactions and the occurrence of nematic phases in liquid crystals, the point-like nature of electrons and their interactions seems at first not to lend itself to the formation of a quantum nematic. So, it was quite a surprise when experiments, first in ultraclean quantum Hall systems [1] and later in $\mathrm{Sr}_{3} \mathrm{Ru}_{2} \mathrm{O}_{7}$ [2], indicated the presence of an electronic nematic phase.

\footnotetext{
*Regroupement Québécois sur les Matériaux de Pointe (RQMP).
}

Strong electronic correlations are believed to be at the source of theses exotic electronic liquids, as theoretically first predicted for the case of a doped two-dimensional Mott insulator [3] due to the melting of a striped phase, and later for quantum Hall systems [4]. Quantum nematics were also discovered in the high-temperature cuprate superconductors [5-9], as well as in the iron arsenide superconductors [10-12], where the relation between the nematic order and superconductivity, and its relation to the close-by structural instability are hotly debated. Pomeranchuk was the first to describe the mechanism by which a Fermi surface can spontaneously break the rotational symmetry [13].

This mechanism has been invoked in the case of the cuprate superconductors [14,15], and it may also play a role in the iron arsenides [10]. Nematic order is also found in CeRhIn a heavy fermion superconductor, where it is most likely related to a spin texture [16]. Nematicity found in $\mathrm{CeB}_{6}$ [17] suggests that a nematic state can be observed in $3 \mathrm{D}$ materials such as the hexaborides, and is not only linked to $2 \mathrm{D}$ and 
quasi-2D systems, opening possibilities for more complex quantum materials. Here, we describe a novel type of nematic order in the hexaboride $\mathrm{EuB}_{6}$, which due to CMR effects associated with the nematic phase carries the potential to play a new role in spintronics. In spintronics, the spin degrees of freedom are used to obtain transistor action, leading to the promise of a lower energy consumption and the unification of storage and processing components.

The interplay between the electronic and magnetic properties of $\mathrm{EuB}_{6}$ are still the subject of controversy despite its simple cubic crystal structure $(P m \overline{3} m)$. EuB 6 has a very low charge carrier density $[18,19]\left(\approx 10^{19} \mathrm{~cm}^{-3}\right)$, these carriers couple to localized Eu $4 f$ moments, which are pure spin with $S=7 / 2$, but whether it should be considered as a semimetal or semiconductor is still an ongoing debate [20-23]. $\mathrm{EuB}_{6}$ becomes ferromagnetic at $T_{\mathrm{C}}=12.6 \mathrm{~K}$, accompanied by an order of magnitude reduction in resistivity and CMR [24] in the vicinity of $T_{\mathrm{C}}$. The specific heat of $\mathrm{EuB}_{6}$ shows an additional anomaly at $T_{M}=15.5 \mathrm{~K}$ [24-26]. This temperature coincides with the first anomaly in the electrical resistivity. In the literature, this anomaly in the electrical resistivity is typically associated with the percolation transition of magnetic polarons. At this temperature, the polarons begin to overlap, releasing the trapped charge carriers, which consequently lowers the resistivity [27].

Magnetic polarons are expected to be important in $\mathrm{EuB}_{6}$ due to its low carrier density and they were indirectly indicated by a number of experiments [26,28-33]. Heuristically, magnetic polarons are composite objects that form when charge carriers polarize a puddle of local moments and become trapped in that puddle [34]. This mechanism is thought to be behind the large CMR effect in $\mathrm{EuB}_{6}$. Since the merger of these polarons leads to a substantially enhanced charge mobility, magnetic polarons in $\mathrm{EuB}_{6}$ were directly identified through a small angle neutron scattering (SANS) experiment [35], while scanning tunneling microscopy (STM) shows that $\mathrm{EuB}_{6}$ is electronically inhomogeneous in the same temperature region [27]. True ferromagnetic order, however, is established only at $T_{\mathrm{C}}[24,36]$. Neutron diffraction on a single crystal of boron ${ }^{11} \mathrm{~B}$ enriched $\mathrm{EuB}_{6}$ oriented along (001) showed (100) and (110) peaks [36]. This is consistent with a propagation vector of (000). A group theoretical analysis using ISOTROPY [37] for this space group and propagation vector suggests a magnetic space group, which is lower than the original $\operatorname{Pm} \overline{3} m$, as pointed out by Süllow and collaborators [26]. This scenario with a transition in two steps is supported by various experimental techniques, such as resistivity and magnetization measurements [24,38], nonlinear Hall effect [30], or muon-spin rotation [29]. These previous experiments all show a linear upward trend of the phase boundary for the phase diagram due to magnetic polarons. In particular, the SANS experiment provided clear evidence for the presence of magnetic polarons in $\mathrm{EuB}_{6}[35]$.

In this paper, we use angle-dependent magnetoresistance oscillations (AMRO) to map out the quantum nematic phase in $\mathrm{EuB}_{6}$ and show that it exists in the region of the phase diagram where magnetic polarons are observed, just below the phase line $T_{\mathrm{QN}}$ in the $(H, T)$ phase diagram, as indicated by magnetostriction measurements [32] and by our measurements of the complex part $\chi^{\prime \prime}$ of the magnetic AC susceptibility $\chi_{\mathrm{AC}}=\chi^{\prime}-i \chi^{\prime \prime}$.

The AMRO technique has been previously used with particular success in the case of metals with two-dimensional Fermi surfaces, such as organic conductors [39,40] and $\mathrm{Sr}_{2} \mathrm{RuO}_{4}$ [41]. Later, this technique was successfully used to demonstrate the presence of a Fermi surface in the overdoped high-temperature superconductor $\mathrm{Tl}_{2} \mathrm{Ba}_{2} \mathrm{CuO}_{6+\delta}$ [42], as well as in $\mathrm{YBa}_{2} \mathrm{Cu}_{3} \mathrm{O}_{6.58}$ [9]. As the Fermi surface of most materials is not spherical, including the one of $\mathrm{EuB}_{6}$, the electrons no longer move on circular orbits. This leads to an angular dependence of the magnetoresistance and consequently to angle-dependent oscillations in the magnetoresistance. In $\mathrm{EuB}_{6}$ previous studies showed a fourfold AMRO pattern at high magnetic fields [43]. A twofold AMRO pattern breaking the axis symmetry was subsequently reported by Glushkov et al. [44], however this was attributed to demagnetization effects. Here, measuring multiple samples allowed us to separate the demagnetization effects from the AMRO signal, and to definitively determine the presence of an additional AMRO component, which suggests the presence of a quantum nematic in $\mathrm{EuB}_{6}$.

\section{METHODS}

The $\mathrm{EuB}_{6}$ single crystals used in our study were grown by the same method as previously used for the sample in Refs. [24-26]. Single crystals were flux-grown with a ratio of $14 \mathrm{mg}$ of $\mathrm{EuB}_{6}$ powder per gram of $\mathrm{Al}$ flux. The mixture was heated in an $\mathrm{Al}_{2} \mathrm{O}_{3}$ crucible using a vertical tube furnace to $1500{ }^{\circ} \mathrm{C}$ at a rate of $200{ }^{\circ} \mathrm{C}$ /hour in a flow of high purity Ar. The mixture was held at that temperature for 10 hours and then cooled down to $1000{ }^{\circ} \mathrm{C}$ at a rate of $5^{\circ} \mathrm{C}$ /hour [45]. The crystals were separated from the $\mathrm{Al}$ flux in boiling sodium hydroxide.

Specific heat $C_{p}$ was measured from 0.4 to $30 \mathrm{~K}$ using a Quantum Design Physical Properties Measurement System (PPMS) with a ${ }^{3} \mathrm{He}$ insert. Magnetic susceptibility was measured using a Quantum Design PPMS AC susceptibility option. The AC susceptibility $\chi_{\mathrm{AC}}$ is defined by $\chi_{\mathrm{AC}}=\chi^{\prime}-i \chi^{\prime \prime}$. For these measurements, the field was applied along [111] with an oscillating component of $5 \mathrm{Oe}$ at a frequency of $77 \mathrm{~Hz}$.

Resistivity was measured for temperatures between 1.8 and $300 \mathrm{~K}$ and magnetic fields between 0 and $9 \mathrm{~T}$ with a PPMS rotator using four-point contact with spot-welded gold wires. Measurements of angle-dependent magnetoresistance oscillations (AMRO) were made by rotating the applied magnetic field $H$ in a plane perpendicular to the current. Measurements were made up to $\mu_{0} H=2 \mathrm{~T}$. At the measured temperatures, no hysteresis was observed. The experiment was done on multiple samples with different cross sections, and aspect ratios. A list of the samples and their dimensions is given in Table I. An image of the main sample \#1 is shown in the Appendix (Fig. 7).

For a sample with a rectangular cross section perpendicular to the current direction, rotation of the applied magnetic field $H$ in the plane perpendicular to the current leads to a change in the induction $B$ due to demagnetization effects. This effect appears even though the magnitude of $H$ is constant and gives rise to a twofold AMRO contribution $a_{\mathrm{dem}}$. 
TABLE I. The demagnetization factors for the measured samples. The second, third, and fourth columns list the sample dimensions, where $L$ is the length along [100], $t$ the thickness along [001], and $w$ the width along [010]. The fourth column is the ratio between the demagnetization factor along the two different directions $\mathscr{D}_{010} / \mathscr{D}_{001}$. The sixth column is the difference in internal field between the [001] and [010] directions for $H=0.5 \mathrm{~T}$ and $T=15 \mathrm{~K}$.

\begin{tabular}{llcccr}
\hline \hline $\begin{array}{l}\text { Sample } \\
\#\end{array}$ & $\begin{array}{c}L \\
(\mathrm{~mm})\end{array}$ & $\begin{array}{c}t \\
(\mathrm{~mm})\end{array}$ & $\begin{array}{c}w \\
(\mathrm{~mm})\end{array}$ & $\mathscr{D}_{010} / \mathscr{D}_{001}$ & $\begin{array}{c}B_{001}-B_{010} \\
(\mathrm{mT})\end{array}$ \\
\hline 1 & 2.413 & 0.428 & 0.437 & 0.983 & -6.3 \\
2 & 2.040 & 0.590 & 0.562 & 1.046 & 15.5 \\
3 & 7.89 & 0.514 & 0.550 & 0.94 & -23.8 \\
4 & 4.32 & 0.650 & 0.507 & 1.25 & 85.9 \\
5 & 5.090 & 0.459 & 0.648 & 0.73 & -119.2 \\
6 & 1.47 & 0.143 & 0.866 & 0.19 & -501.9 \\
\hline \hline
\end{tabular}

\section{RESULTS AND DISCUSSION}

We start the discussion of our results by presenting the AMRO measurements on the sample with an almost square cross section (sample \#1 in Table I) in Fig. 1, where the demagnetization effects are smallest. The geometry used in the measurement is schematically shown in Fig. 1(a). In our convention, we define the angle $\theta=0$ when the field is applied along [001]. The field rotates in the (100)-plane around the direction of the current, which flows parallel to [100]. In order to eliminate any contribution from the Hall effect to the AMRO, we measured the AMRO for both $+H$, and $-H$, and averaged the values.

As can be seen in Fig. 1(c), for this sample the AMRO signal disappears for temperatures above $50 \mathrm{~K}$ in an applied field of $0.5 \mathrm{~T}$. Below $50 \mathrm{~K}$, the strongest AMRO component is a fourfold oscillation $a_{4}$ from the Fermi surface. This contribution is expected from the band structure of $\mathrm{EuB}_{6}$, which has a cubic lattice. Such a fourfold oscillation has previously been reported $[43,44]$. However, for temperatures close to $T_{M}$, where the specific heat shows a first anomaly [24-26], and magnetic fields below $1 \mathrm{~T}$, there are two different twofold contributions the AMRO, which dominate the AMRO response: a smaller twofold component $a_{\mathrm{dem}}$, which is commensurate with the fourfold component $a_{4}$ from the Fermi surface and an $a_{2}$ component, which is shifted with respect to the $a_{4}$, and the $a_{\mathrm{dem}}$ signal. This is particularly visible in the traces taken at $11 \mathrm{~K}$, which were taken in different applied magnetic fields, as shown in Fig. 1. Applied fields above $2 \mathrm{~T}$ almost completely suppress the twofold components, as can be seen in Fig. 1(e) for a temperature of $14 \mathrm{~K}$.
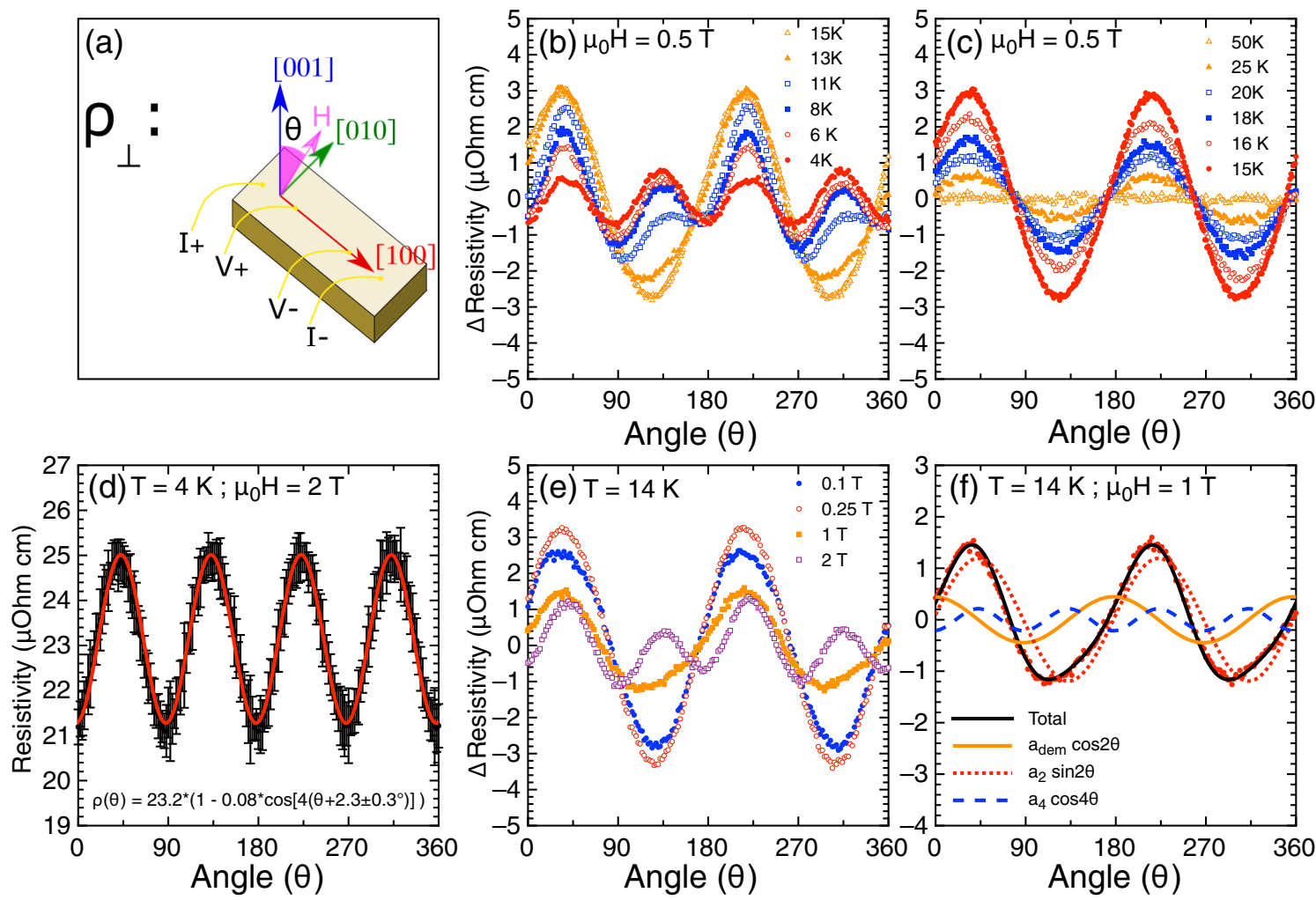

FIG. 1. Angle-dependent magnetoresistance oscillations (AMRO) in a sample of almost square cross section (sample \#1) (a) Schematics of the measurement geometry: for measuring the perpendicular magnetoresistance $\rho_{\perp}$, the applied magnetic field $H$ is rotated by an angle $\theta$ in the (100)-plane from [001] to [010], thus maintaining $H$ perpendicular to the current flowing along [100]. (b) and (c) show the oscillatory component of $\rho_{\perp}-\rho_{0}$ vs the field angle $\theta$ for $\mu_{0} H=0.5 \mathrm{~T}$ at different temperatures. (d) shows the resistivity at $4 \mathrm{~K}$ and $2 \mathrm{~T}$ used to determine the experimental rotator angle $\theta_{0}=-2.3^{\circ}$ at which $H \|$ [001]. The solid line is the fit to a fourfold contribution. This figure also shows the typical noise in our AMRO measurements; these uncertainties were taken into consideration when fitting the AMRO oscillations. (e) Perpendicular magnetoresistance $\rho_{\perp}-\rho_{0}$ vs $\theta$ at $T=14 \mathrm{~K}$ for different applied fields. (f) Comparison of the different AMRO components at $14 \mathrm{~K}$ and $1 \mathrm{~T}$, as described by Eq. (1). 
Thus, a full description of the AMRO requires three components:

$$
\begin{aligned}
\rho(H, T, \theta)= & \rho_{0}(H, T)\left(1+a_{2} \sin 2 \theta+a_{4} \cos 4 \theta\right. \\
& \left.+a_{\mathrm{dem}} \cos 2 \theta\right),
\end{aligned}
$$

where $a_{\mathrm{dem}}$ is due to demagnetization effects when the sample cross section deviates from being square, $a_{4}$ is a fourfold component, and $a_{2}$ a symmetry breaking contribution. This shift result in peak position for the twofold components at roughly $37^{\circ}$. In Eq. (1), all the values are given as a fraction of the mean resistivity value $\rho_{0}(T, H)$.

In order to find the correct mechanical zero of the rotator, we first measured the AMRO at $4 \mathrm{~K}$ and in a field of $2 \mathrm{~T}$ shown in Fig. 1(d), where the twofold oscillations are strongly suppressed. We then fitted this set of data to

$$
\rho(H, T, \theta)=\rho_{0}(H, T)\left(1+a_{4} \cos (4(\theta+\gamma))\right),
$$

which allowed us to determine the mechanical offset $\gamma$ of $2.3 \pm 0.3^{\circ}$. In the following, we then shifted the origin to this value. A fit of Eq. (1) together with the resulting is shown in Fig. 1(f) for $14 \mathrm{~K}$ in a field of $1 \mathrm{~T}$ for sample \#1. We then proceeded to extract the amplitudes for the three components to the AMRO by fitting Eq. (1) for sample \#1 in Table I. The results are shown in Fig. 2. The signal from the Fermi surface $a_{4}$ starts to pick up strength below $20 \mathrm{~K}$. Its strength increases continuously with decreasing temperature and increasing field. However, the symmetry breaking contribution $a_{2}$ to the AMRO goes through a maximum as a function of decreasing temperature. This signal starts to pick up in strength below $20 \mathrm{~K}$ then goes through a maximum, which is roughly centered at $T_{\mathrm{C}}$ of $12.6 \mathrm{~K}$, and then becomes weaker toward the lowest measured temperatures. The symmetry breaking contribution $a_{2}$ also goes through a maximum of increasing field. $a_{2}$ is strongest for $0.25 \mathrm{~T}$ but decreases for higher fields. A detailed discussion of the demagnetization contribution $a_{\mathrm{dem}}$ is given in the Appendix.

\section{A. Fermi surface contribution $a_{4}$ to the AMRO}

More interesting are the $a_{4}$ and $a_{2}$ components to the AMRO. First we are going to discuss the $a_{4}$ component, which is due to the fact that $\mathrm{EuB}_{6}$ has an anisotropic Fermi surface, as shown in Fig. 3. This finding is in agreement with the results of a previous AMRO study on $\mathrm{EuB}_{6}$ [43], where the same rotation axis was used. Conventional metals possess a three-dimensional Fermi surface, which is the position of the long-lived electronic excitations in reciprocal space, which determine their electronic properties at low temperatures [42]. These excitations, as well as the geometry of Fermi surface can be probed by measuring the AMRO. The AMRO oscillations can be calculated using the Shockley-Chambers tube integral form of the Boltzmann transport equation, where we have assumed an isotropic mean-free-path [47].

The Fermi surface of $\mathrm{EuB}_{6}$ was previously observed by de Haas-van Alphen (dHvA) measurements at $0.4 \mathrm{~K}$ [46]. The experimentally observed Fermi surface has a cubic symmetry with symmetric ellipsoids at the $X$ points of the Brillouin zone (see to the right of Fig. 3). A similar Fermi surface was also seen in angle resolved photoemission (ARPES) [48], which agrees well with band structure calculations [20]. As the
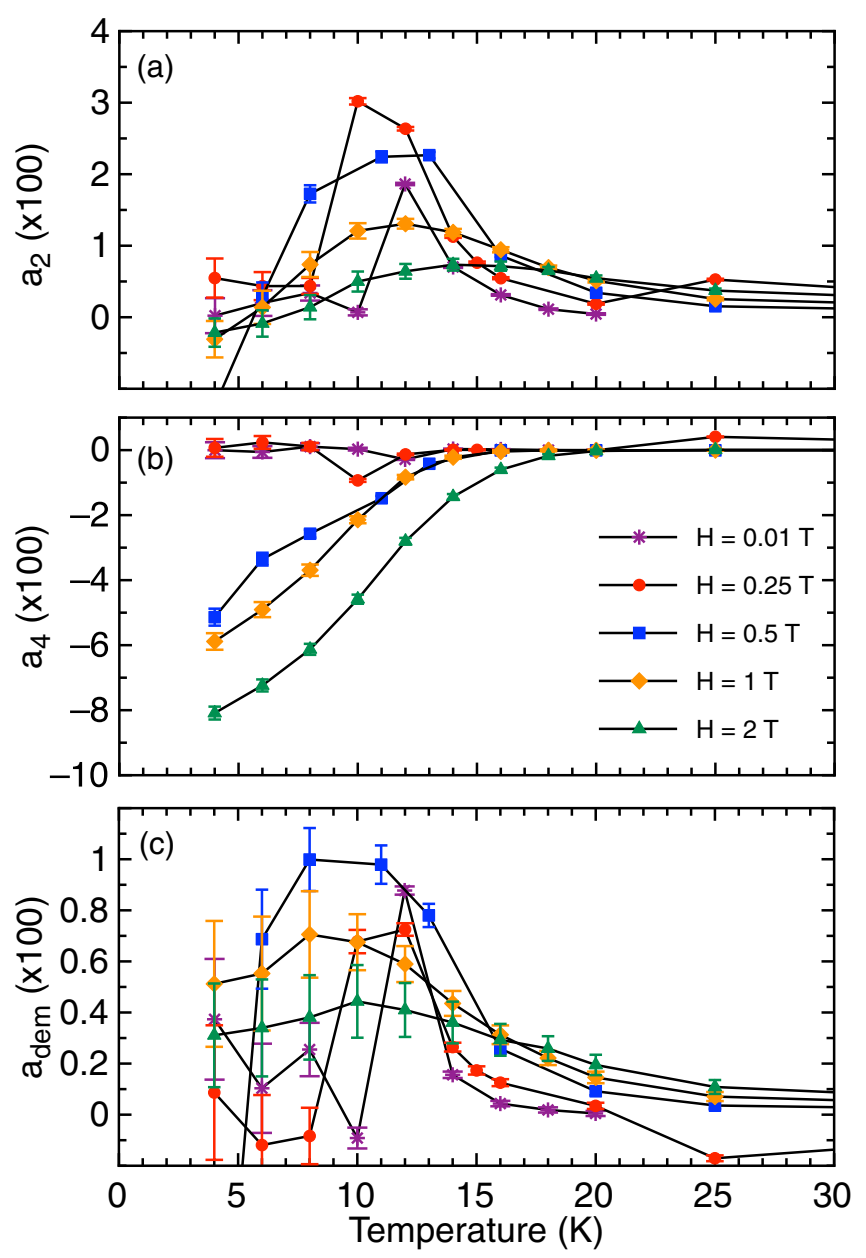

FIG. 2. Amplitude of the different AMRO components vs the temperature. (a) Amplitude $a_{2}$ of the twofold symmetry breaking components, (b) amplitude $a_{4}$ of the fourfold component due to the shape of the Fermi surface, and (c) amplitude $a_{\text {dem }}$ of the demagnetization for applied magnetic fields of $0.01 \mathrm{~T}$ (purple stars), $0.25 \mathrm{~T}$ (red circles), $0.5 \mathrm{~T}$ (blue square), $1 \mathrm{~T}$ (orange diamonds), and $2 \mathrm{~T}$ (green triangles). This data was taken on sample \#1 in Table I.
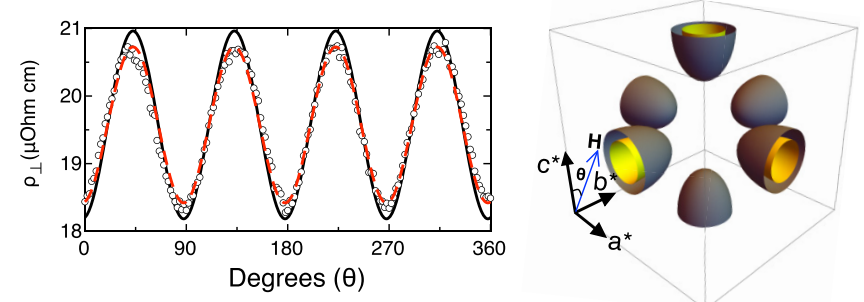

FIG. 3. (Left) The angular dependence of the resistivity tensor in a magnetic field of $1 \mathrm{~T}$ as calculated from Eq. (3) is illustrated by the black line. The data are taken from sample \#1 at $4 \mathrm{~K}$ and $1 \mathrm{~T}$ and fitted with Eq. (1) (red-dash line). (Right) The Fermi surface of $\mathrm{EuB}_{6}$, as observed by dHvA [46]. The gray outer ellipsoids show the electron pockets and the yellow inner ellipsoids the holes. There are a total of six half ellipsoids in one unit cell for both electrons and holes. 

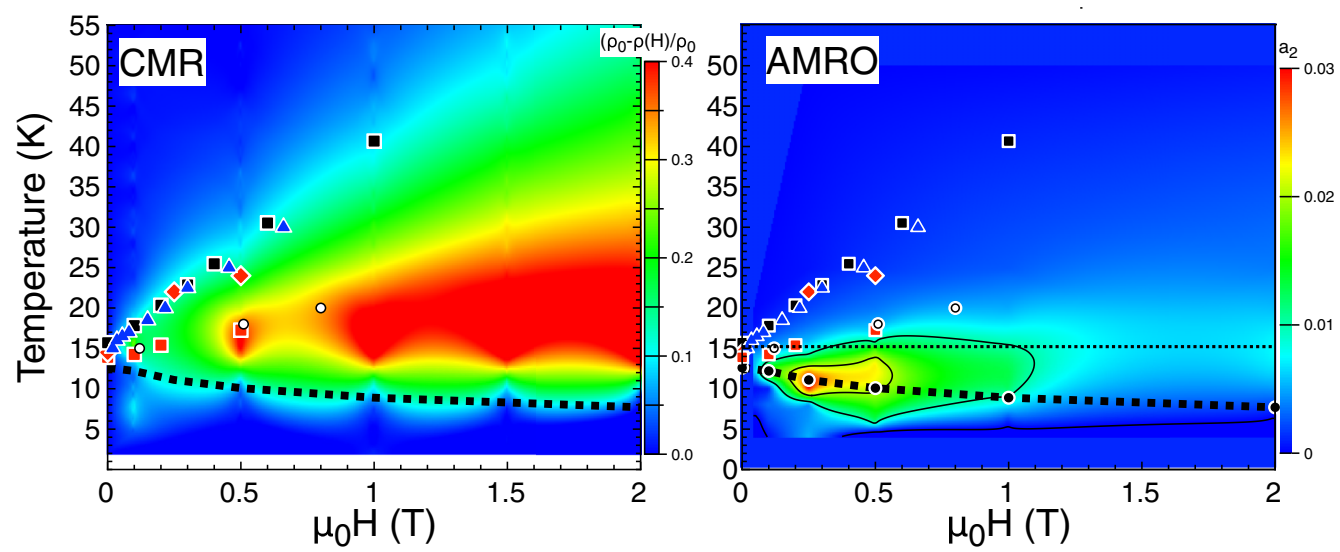

FIG. 4. CMR and AMRO phase diagram. (Full-black squares) Hall effect measurements [30], (red diamonds) small-angle neutron scattering measurements [35], (teal triangles) magnetoresistance peak [31], (open-black circles) magnetostriction [32], (red squares) $T_{M}$ taken from $\chi^{\prime \prime}$ measurements (Fig. 6). (Black circles) $T_{\mathrm{C}}$ from heat capacity found using a fit to mean field theory (Appendix Fig. 8). Left: CMR phase diagram with $\left(\rho_{0}-\rho_{001}(H)\right) / \rho_{0}$ vs temperature, and magnetic fields, where $\rho_{0}$ is the electrical resistivity in zero field. Right: AMRO phase diagram with the amplitude of the two contributions vs temperature and field. Magnetic polarons are found in the region below the line, which is delineated by Hall effect and SANS measurements, and above the ferromagnetic transition line.

dHvA measurements did not indicate a splitting of the Fermi surface, and tunneling experiments indicate a spin-spitting of the Fermi surface only in the ferromagnetic state, we did not take spin-splitting into consideration in our modeling.

The Fermi surface of $\mathrm{EuB}_{6}$ has an electron pocket radius ratio of 1.8 , and a ratio of 1.6 for the holes. The ARPES data [48] allows us to estimate the radius of $0.1 \AA^{-1}$ for the ellipsoids. The temperature dependence of the amplitudes of the dHvA oscillations [18] gives an effective mass of (0.225 \pm $0.01) m_{e}$ for the electrons and $(0.313 \pm 0.02) m_{e}$ for the holes. Here, $m_{e}$ is the electron mass. Using all these parameters, we can calculate the conductivity tensor when a field is applied to the system through the Chambers formula $[49,50]$ :

$$
\begin{aligned}
\sigma_{i j}= & \sum_{\alpha} \frac{e^{2}}{4 \pi^{3}} \frac{m_{\alpha}^{*}}{\hbar^{2} k_{F, \alpha}} \int_{S_{\alpha}} v_{i, \alpha}(\mathbf{k}, 0) d^{2} \mathbf{k} \\
& \times \int_{0}^{\infty} v_{j, \alpha}(\mathbf{k}, t) e^{-t / \tau} d t
\end{aligned}
$$

where $S_{\alpha}$ is the Fermi surface sheet associated with band $\alpha$, $k_{F, \alpha}$ is the Fermi momentum of band $\alpha$ defined as $k_{F, \alpha}=$ $\sqrt[3]{3 \pi^{2} n_{\alpha}}$, where $n_{\alpha}$ is the corresponding charge carrier density. The sum is over all occupied bands $\alpha$, where $v_{i, \alpha}$ is the velocity component of band $\alpha$ (either electron or hole), and $\tau$ is the quasiparticle lifetime.

In $\mathrm{EuB}_{6}$, there is no anisotropy in the quasiparticle lifetime, unlike what is observed in some cuprates [9]. Also, for $\mathrm{EuB}_{6}$, the product of cyclotron frequency $\omega_{c}$ and $\tau$ is greater than one, therefore the quasiparticles on the Fermi surface complete at least one orbit before they scatter [42]. This removes some of the complexity in fitting, which affects the shape of the AMRO oscillations. Furthermore, since the Fermi pockets in $\mathrm{EuB}_{6}$ are far from the Brillouin zone boundaries, we can neglect large variation in the density of states due to a van Hove singularity [51].

We performed a theoretical modeling to gain further insight into our AMRO observations by using the relaxation time approximation for the conductivity. AMRO has been instru- mental in the early understanding of the band structure of metals [52,53]. More recently for example, it provided clear evidence for a nematic state and a symmetry-breaking of the Fermi surface in a high- $T_{c}$ superconductor [9]. From Eq. (3), a fourfold oscillation in $\rho_{\perp}$ is expected when the magnetic field is rotated in the $b-c$ plane (see Fig. 3) due to the $C_{4}$ symmetry of the Fermi surface. The calculation at a applied field of $1 \mathrm{~T}$ gives an amplitude $a_{4}$ of -0.07 , which is lower than the amplitude of -0.06 found at $4 \mathrm{~K}$. This is valid since the amplitude keep decreasing with temperature (see Fig. 2), and the temperature in the calculations was zero. The resulting fourfold oscillatory component is shown in Fig. 3. The overall constant resistivity differs strongly with the experimental data, which is due mainly by approximation and initial parameters. The value was scaled in Fig. 3 to make the comparison. Further improvement to the numerical calculation will be done to investigate the symmetry breaking.

\section{B. Symmetry breaking contribution $a_{2}$ to the AMRO}

Finally, we will discuss the new symmetry breaking contribution $a_{2}$. The symmetry breaking twofold contribution is not due to aluminum inclusion from the flux growth, as those would contribute a fourfold pattern, which is in phase with the $a_{4}$ component of the AMRO as discussed in further detail in Appendix B. The $a_{2}$ contribution breaks the inversion symmetry, which is in agreement with the spin-split Fermi surface observed in a recent ARPES study [54]. This breaking of symmetry would also suggest that we are observing a chiral quantum nematic. A transition to a Fermi surface with a chiral superstructure (see Ref. [55], and references therein) was found in theoretical attempts to explain the ferromagnetism in La doped $\mathrm{CaB}_{6}$ [55-57]. However, later it was experimentally determined that the ferromagnetism in $\mathrm{La}$ doped $\mathrm{CaB}_{6}[58]$ is rather due to extrinsic impurities [59,60]. This mechanism, requires a semi-metallic band structure, which in semimetallic or small gap divalent hexaborides depends on the distance between the boron cages in neighboring unit cells 


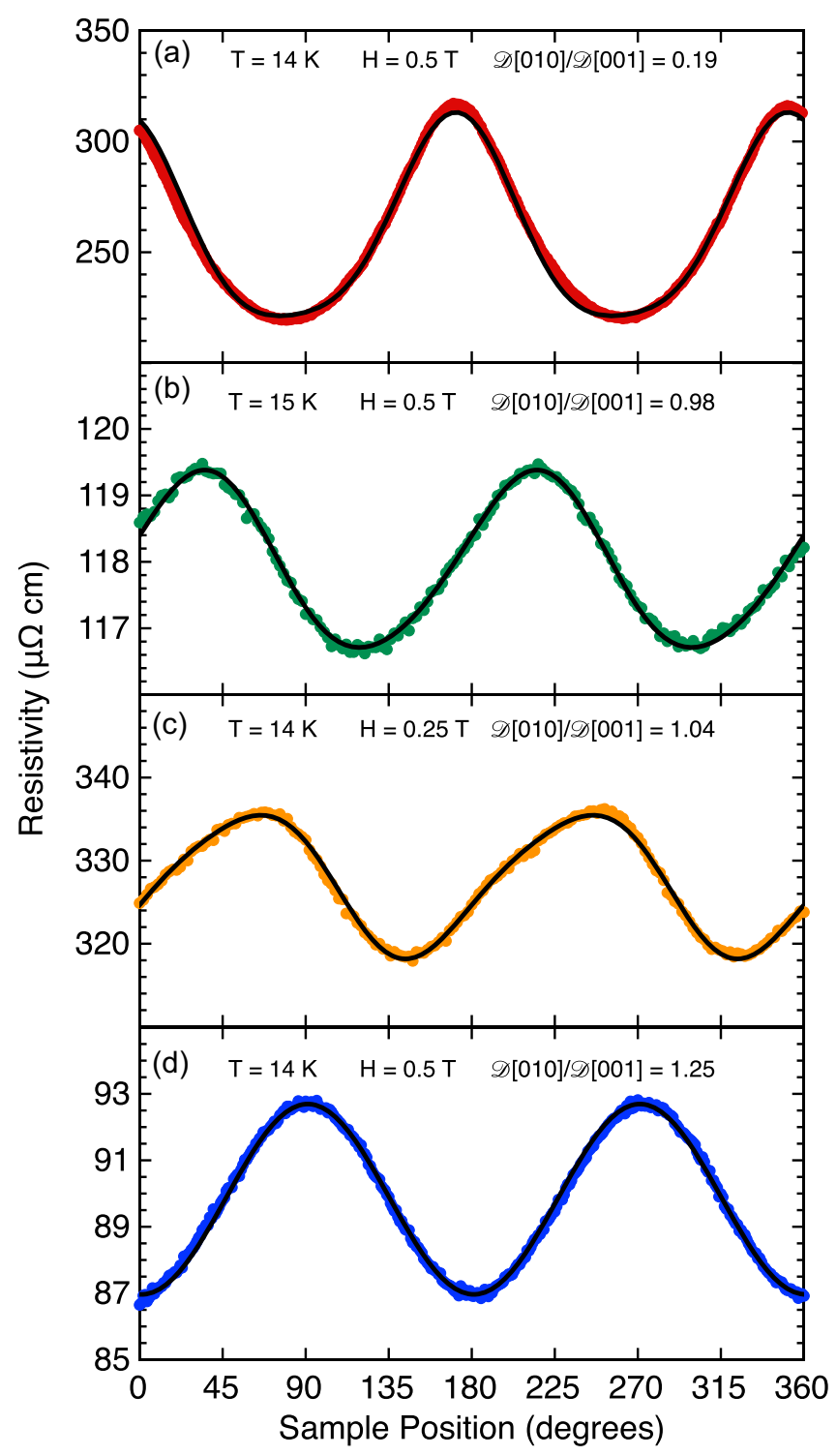

FIG. 5. Comparison of the AMRO signal around $\mathrm{T}_{\mathrm{M}}$ and at applied fields of 0.25 , and $0.5 \mathrm{~T}$ for (a) sample \#6, (b) sample \#1, (c) sample \#2, and (d) sample \#4. The black lines are a fit to Eq. (1).

[20-23]. Under the influence of electron-electron interaction these systems show the tendency to form a helical superstructure [55]. However, it is not possible to reconcile a transition from this twofold symmetry to a higher fourfold symmetry as sequence of second order phase transitions, as a second order phase transition has to always lower the symmetry.

The colors in Fig. 4 show the strength of the symmetry breaking component $a_{2}$ of the AMRO, as determined from the size of the twofold contribution to $\rho_{\perp}$ varying as $\sin (2 \theta)$, where $\theta$ is the angle of the applied field in the plane perpendicular to the current [Fig. 1(a)]. The symmetry breaking component appears below the $T_{M}$ line in the phase diagram demarked by magnetostriction [32] and $\chi^{\prime \prime}$ given in Sec. III C. Also, this signal is only seen in nearly cubic samples. Figure 5 shows how noncubic samples deviate from the $\sin 2 \theta$ because of demagnetization. This effect is explained in more detail in Appendix A.

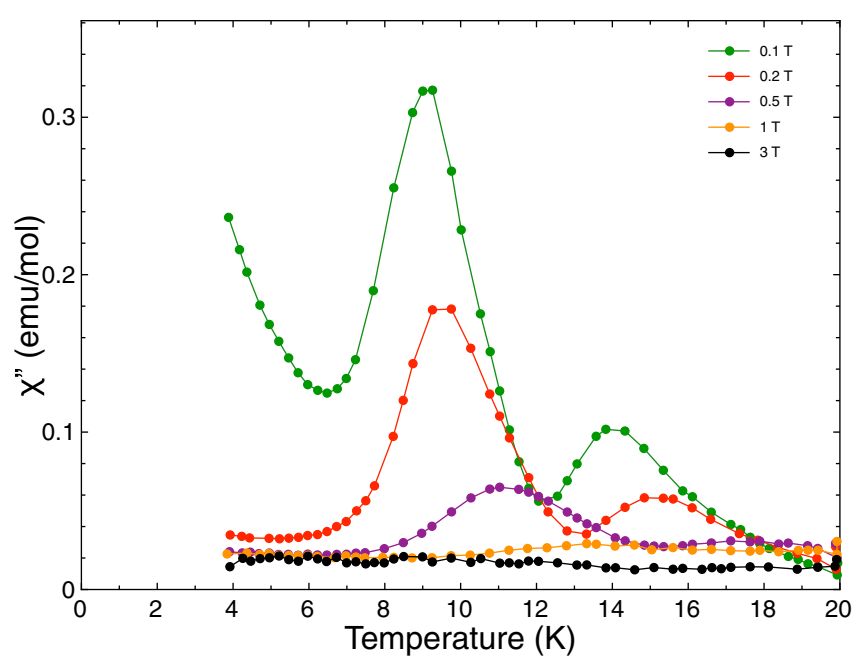

FIG. 6. Imaginary part of the magnetic susceptibility. $\chi^{\prime \prime}$ vs temperature for different applied fields. The second maximum in $\chi^{\prime \prime}$ coincides with where the AMRO is strongest.

\section{Magnetic susceptibility}

In order to better map out the $(H, T)$ phase diagram, and delineate the region where we observe a quantum nematic in the AMRO, we carried out the AC susceptibility. The AC susceptibility is a complex value and reads $\chi_{\mathrm{AC}}=\chi^{\prime}-i \chi^{\prime \prime}$, where the real component $\chi^{\prime}$, related to the reversible magnetization process, and the imaginary component $\chi^{\prime \prime}$ is related to losses due to the irreversible magnetization process and energy absorbed from the field [61]. One such example are the vortices in type-II superconductors, which are topological defects of an homogeneous order parameter.

Figure 6 shows the second order magnetic susceptibility $\chi^{\prime \prime}$. The peak in $\chi^{\prime \prime}$ at the lower temperature corresponds to the ferromagnetic transition, while the peak at higher temperatures corresponds to where the signal of magnetic polarons is strongest. The positions of the high-temperature peaks are shown as red squares in Fig. 4. This phase boundary coincides with the increase in anisotropic resistivity signal, which suggests a strong connection between nematicity and magnetic polarons in $\mathrm{EuB}_{6}$.

\section{OUTLOOK}

The role electronic phase separation and magnetic polarons $[3,62]$ play in high-temperature superconductivity clearly motivates the need for a model system for studying magnetic polarons. In $\mathrm{EuB}_{6}$, the regions of the $(H, T)$ phase diagram where SANS indicates magnetic polarons and where AMRO displays quantum nematicity coincide, providing strong evidence that both nematicity and magnetic polarons originate from the same electronic correlations. This makes our results on $\mathrm{EuB}_{6}$ particularly important, as unlike the hightemperature superconductors, $\mathrm{EuB}_{6}$ possess a high degree of structural order without a nearby lattice instability [63]. $\mathrm{EuB}_{6}$ is thus an excellent model system to study quantum nematicity. Further, the presence of both an electronic nematic and colossal magnetoresistance suggests that $\mathrm{EuB}_{6}$ can be used as a novel platform for spintronic devices [64]. This promises 
a way to harness strong electronic correlations for spintronic applications, and motivates the search for other materials with magnetic polarons.

\section{ACKNOWLEDGMENTS}

The research at Université de Montréal received support from the Natural Sciences and Engineering Research Council of Canada (Canada). W.W.-K. was in addition supported by the Fondation Courtois, a Canada Research Chair, and a "Établissement de nouveaux chercheurs et de nouvelles chercheuses universitaires" Grant from the FRQNT. The work at the MPI-CPFS was enabled through a DAAD Grant. The authors would like to thank Prof. S. Süllow from the TU Braunschweig in Germany for sharing his magnetoresistance data on $\mathrm{EuB}_{6}$ with us. We would also like to thank Nicolas Gauthier for his advice on magnetic structures.

\section{APPENDIX A: DEMAGNETIZATION CONTRIBUTION TO THE AMRO}

We carried out magnetoresistance measurements on an EuB $_{6}$ sample with an almost square cross section (sample \#1 in Table I, with $\mathscr{D}_{010} / \mathscr{D}_{001}=0.983$, as shown in Fig. 7). The demagnetization contribution can be calculated, if we know the magnetoresistance as a function of the magnetization (see for example [26]), using the formula for the demagnetization factor [68], we can then calculate this contribution. The contribution $a_{\mathrm{dem}}$ from the demagnetization to the AMRO depends on both the internal magnetic field $B$ and the magnetoresistance $\frac{\partial \rho}{\partial B}$. The demagnetizing factor $\mathscr{D}$ for rectangular ferromagnetic prisms was given by [68]. If for example the sample does not have a square cross section [for example, $t([001])<w([010])$, as shown in Fig. 1(a)], then if $\mathscr{D}_{001}>$ $\mathscr{D}_{010}$, rotating the sample around the direction of the current flow ([100]) from the [001] direction to the [010] direction is equivalent to an increase in the magnetic field $\left(\Delta B=B_{001}-\right.$ $\left.B_{010}<0\right)$. As the magnetoresistance $\frac{\partial \rho}{\partial B}$ is negative, the effect of demagnetization would be that $\Delta \rho_{\text {demag }} \approx \frac{\partial \rho}{\partial B} \Delta B>0$. Thus for such a sample, the $\rho(B \|[001])>\rho(B \|[010])$.

For a given direction, the internal field in a sample is defined as [69]:

$$
\begin{aligned}
B / \mu_{0} & =H_{0}+M-H_{\mathscr{D}} \\
& =H_{0}+M-\mathscr{D} M
\end{aligned}
$$
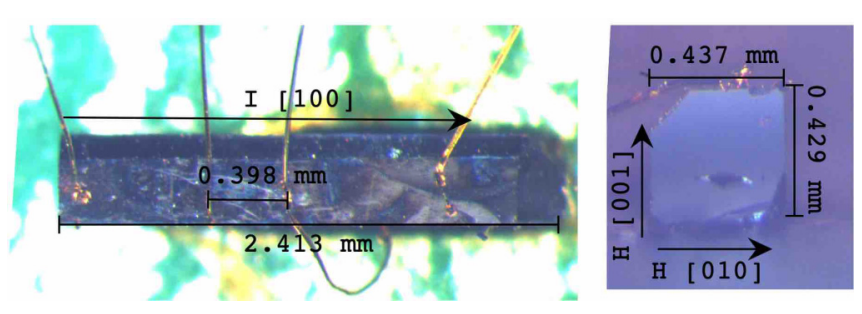

FIG. 7. Dimensions of sample \#1. For this sample, the demagnetization factors are $\mathscr{D}_{100}=0.080, D_{010}=0.46$, and $\mathscr{D}_{001}=0.46$. For the contacts we spot-welded gold wires with a diameter of $25 \mu \mathrm{m}$ to the sample.

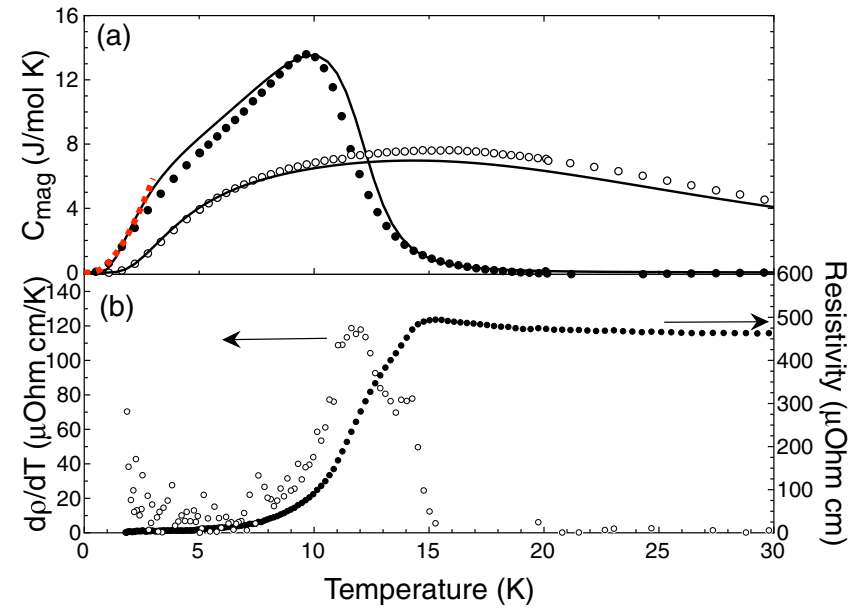

FIG. 8. Specific heat and electrical resistivity of $\mathrm{EuB}_{6}$. (a) Magnetic specific heat capacity $C_{M}=C_{p}-C_{\mathrm{ph}}$ after subtraction of the phonon contribution $C_{\mathrm{ph}}$ of $\mathrm{EuB}_{6}$ at zero field. The curves correspond to $0 \mathrm{~T}$ (full circles) and $5 \mathrm{~T}$ (empty circles). The black line is a fit to the mean field model for the heat capacity [65]. A field of $0.1 \mathrm{~T}$ was used for the zero field data. The red dashed line is a fit to a spin wave contribution, which is proportional to $T^{3 / 2} e^{-\Delta / T}$ [66]. It gives a gap of $\Delta=1.11 \pm 0.03 \mathrm{~K}$ similar to the one reported by NMR techniques [67]. Contrary to previous work [25], we only see a shoulder at the onset of $T_{\mathrm{M}}$ instead of a second peak in $C_{M}$. (b) Electrical resistivity $\rho$ at zero field, and its temperature derivative $\partial \rho / \partial T$ vs temperature for the sample used in the main text.

$$
=H_{0}+(1-\mathscr{D}) M\left(T, H, H_{\mathscr{D}}\right) \quad,
$$

Here, $H_{0}$ is the applied field and $M$ is the magnetization as a function of $H$ and $H_{\mathscr{D}}$. Here we assumed a uniform magnetization in our samples, which are cuboids, whereas strictly speaking, the magnetization is only uniform for ellipsoids. The difference in the internal magnetic field, for when the magnetic field is applied along a different geometric axis, can then be calculated using demagnetization factor [68]. If we know the magnetization for a given applied field, we can then compute the internal field, which is the sum of the applied magnetic field plus the induced magnetization corrected by the demagnetization. We can find the induced magnetization for a given applied field with the help of Eq. (A3), using a mean field approximation [65]. The use of a mean field approximation for finding the magnetization is justified, since it describes the field dependence of the specific heat well, as is shown in Fig. 8.

To find the magnetization, we numerically solve the following set of equations [65]:

$$
\begin{gathered}
M=M_{0} B_{J}(x), \\
B_{J}(x)=\frac{2 J+1}{2 J} \operatorname{coth}\left(\frac{2 J+1}{2 J} x\right)-\frac{1}{2 J} \operatorname{coth}\left(\frac{x}{2 J}\right), \\
x=\frac{g J \mu_{\mathrm{B}}}{k_{\mathrm{B}} T}\left(\mu_{0} H_{0}+(\lambda-\mathscr{D}) \mu_{0} M\right), \\
\lambda=\frac{3 k_{\mathrm{B}} T_{\mathrm{C}}}{N g^{2} \mu_{\mathrm{B}}^{2} \mu_{0} J(J+1)} .
\end{gathered}
$$




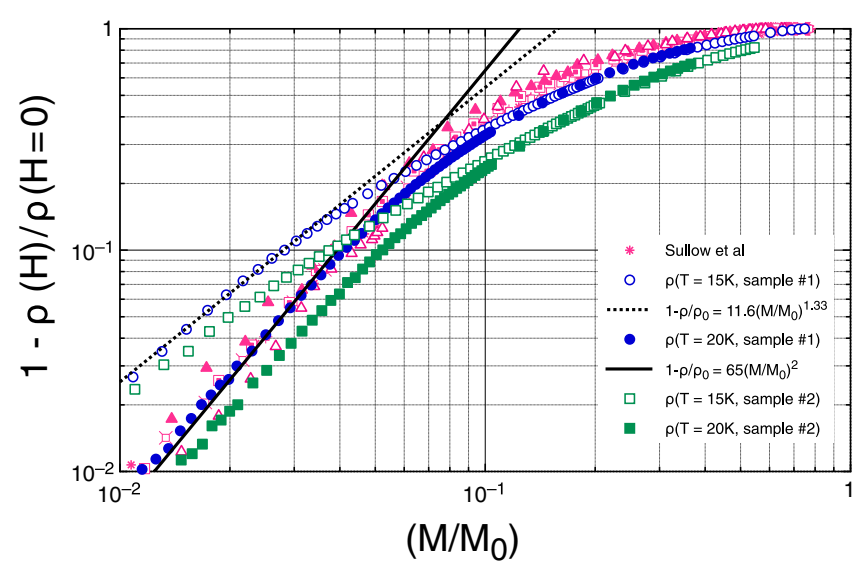

FIG. 9. Magnetoresistance vs magnetization. Here, $M_{0}$ is the value for the saturation magnetization of $\mathrm{EuB}_{6}$ and $\rho(H)$ the electrical resistivity in an applied field of $H$. The blue circles show the magnetoresistance of the sample with a square cross section (sample $\# 1)$ as $1-\rho(H) / \rho(H=0)$ at $15 \mathrm{~K}$ (open), and at $20 \mathrm{~K}$ (closed). The green squares show the magnetoresistance of the sample with a square cross section (sample $\# 2)$ as $1-\rho(H) / \rho(H=0)$ at 15 $\mathrm{K}$ (open), and at $20 \mathrm{~K}$ (closed). The calculation of $M$ accounts for demagnetization. For all, the magnetic field was applied along [001]. The solid, dotted and dash lines are a fit to equations a power law. The solid pink points are data reported previously by Süllow et al. [70], which follows $1-\rho(H) / \rho(H=0) \sim 75\left(\frac{M}{M_{0}}\right)^{2}$.

Here, $B_{J}(x)$ is the Brillouin function, $J$ is the total angular momentum of $\frac{7}{2}$ of $\mathrm{EuB}_{6}, T_{\mathrm{C}}$ the Curie-Weiss temperature of $12.6 \mathrm{~K}$, the $g$ factor is 2 and $N$ is the number of magnetic atoms per volume. However, in order to be able to calculate the demagnetization contribution $a_{\text {dem }}$, the magnetization dependence of the electrical resistivity has to be measured.

Madjumdar and Littlewood proposed that the magnetoresistance in metallic ferromagnets, and doped magnetic semiconductors should be negative, and proportional to the square field induced magnetization [71]. In their theory, this behavior is due to the suppression of the magnetic fluctuations by the applied magnetic field. Such a behavior was reported by [26], i.e., $1-\frac{\rho(H)}{\rho(H=0)} \sim\left(\frac{M}{M_{0}}\right)^{2}$. The results of these measurements are shown in Fig. 9 for $15 \mathrm{~K}$ and $20 \mathrm{~K}$. For comparison we also show data extracted from [70]. Our data does follow a power law:

$$
\begin{aligned}
& 1-\frac{\rho(H)}{\rho(H=0)}=65\left(\frac{M(H)}{M_{0}}\right)^{2} \text { for } T=20 \mathrm{~K} \\
& 1-\frac{\rho(H)}{\rho(H=0)}=11.6\left(\frac{M(H)}{M_{0}}\right)^{1.33} \text { for } T=15 \mathrm{~K},
\end{aligned}
$$

and agrees with the exponent of two at low field above $T_{\mathrm{M}}$. Using the factor found in Eqs. (A8) and (A9) and the charge carrier concentration, we can determine the position of our measurements in the plot presented by Madjumdar and Littlewood. Our factor $C$ is equal to 65 , our charge carrier concentration is $5 \times 10^{19} \mathrm{~cm}^{-3}$ at low field and temperature of $20 \mathrm{~K}$ and our magnetic correlation length is $4.18 \AA$. The charge carrier concentration was taken from Hall coefficient

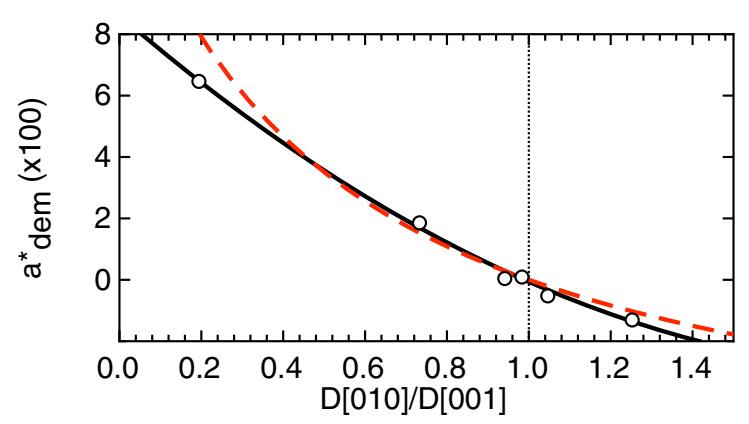

FIG. 10. Twofold contribution to the AMRO. The open circles show the amplitude $a_{\mathrm{dem}}^{*}$ retrieved from the fit of Eq. (1) vs the ration of the demagnetization factors, $\frac{\mathscr{D}_{010}}{\mathscr{D}_{01}}$ for all samples at $15 \mathrm{~K}$ and in an applied field of $0.5 \mathrm{~T}$. The amplitude was normalized as $a_{\mathrm{dem}}^{*}=$ $\frac{\rho_{0}\left(T=15 \mathrm{~K}, H=0.5 \mu_{0} \mathrm{~T}\right)}{\rho_{0}(T=15 \mathrm{~K}, H=0)} a_{\mathrm{dem}}$ for a comparison with the data. The solid line a fit of $a_{\mathrm{dem}} \propto\left(\frac{\mathscr{D}_{010}}{\mathscr{D}_{001}}\right)^{2}$. The dashed line is the calculated amplitude using the demagnetization and the CMR as given by Eq. (A9) as described in the text.

measurements. These values place us in the same region as other $\mathrm{EuB}_{6}$ experiments [70]. The first indication that $a_{\mathrm{dem}}$ is indeed due to demagnetization comes from the fact that it is commensurate with the $a_{4}$ component from the Fermi surface. As this component is linked to the shape of the cross section, and the crystal habit is given by the cubic symmetry of $\mathrm{EuB}_{6}$, this is the expected behavior. We used Eq. (A9) to calculate the twofold AMRO component $a_{\mathrm{dem}}$ due to a combination of demagnetization effects and a large $\mathrm{CMR}$ in $\mathrm{EuB}_{6}$. The result of this calculation is shown in Fig. 10 as the red dashed line for a temperature of $15 \mathrm{~K}$, and in an applied magnetic field of $0.5 \mathrm{~T}$. Here, we calculated $a_{\mathrm{dem}}$ as function of the ratio of the demagnetization factor $\frac{\mathscr{D}_{010}}{\mathscr{D}_{001}}$ using a sample length $L$ of $1 \mathrm{~mm}$.

\section{APPENDIX B: ALUMINUM INCLUSIONS}

Previous interpretations of dHvA Fermi surface measurements of $\mathrm{SmB}_{6}$ were plagued by aluminum inclusions, see Refs. [72,73]. It was found that the aluminum inclusions are epitaxial single crystals co-oriented with the (100) direction $\mathrm{SmB}_{6}$. We would expect the same growth direction for aluminum inclusions in $\mathrm{EuB}_{6}$. Such inclusion, if present, would then lead to fourfold AMRO pattern [74], with the same angle dependence as observed at high temperatures and high magnetic fields in $\mathrm{EuB}_{6}$. Thus, the lowering of the symmetry in the AMRO from fourfold to twofold in $\mathrm{EuB}_{6}$ cannot be explained by the presence of epitaxial aluminum inclusions.

\section{APPENDIX C: AMRO CALCULATIONS}

In Eq. (3) of the main text we calculated a numerical integral over the Fermi surface $S$, which is shown in Fig. 3 of the main text. The total Fermi energy for one closed pocket 
(for example the electron band at position $\mathrm{X}$ ) is [75]:

$$
\begin{gathered}
E_{F}=\frac{V}{(2 \pi)^{3}} \frac{\hbar^{2}}{2 m^{*}} \iiint_{\left\{\frac{k_{x}^{2}}{k_{a}^{2}}+\frac{k_{y}^{2}}{k_{a}^{2}}+\frac{k_{z}^{2}}{k_{b}^{2}} \leqslant 1\right\}} d k_{x} d k_{y} d k_{z} \\
\times\left(k_{x}^{2}+k_{y}^{2}+k_{z}^{2}\right) \\
E_{F} / N=\frac{\hbar^{2}}{2 m^{*}} k_{F}^{2}, \text { where } \\
k_{F}^{2}=\frac{4}{15}\left(2 k_{a}^{2}+k_{b}^{2}\right),
\end{gathered}
$$

where $k_{a}$ is the minor axis and $k_{b}$ is the major axis of the elliptical pockets. These axes are linked to the volume of the Fermi volume, and hence, to the charge carrier density. So, we can obtain the minor axis from the ratio of the radii $R^{*}=k_{b} / k_{a}$, and the density of charge carriers $n$ as

$$
k_{a}=\frac{\sqrt[3]{3 \pi^{2} n}}{\sqrt{\frac{4}{15}\left(2+R^{* 2}\right)}}
$$

Here, $n$ and $R^{*}$ will be different for the two bands (electrons and holes). The velocity of the charge carriers was calculated as follows:

$$
\mathbf{v}(\mathbf{k})=\frac{1}{\hbar} \nabla_{\mathbf{k}} E(\mathbf{k})
$$

and

$$
E(\mathbf{k})=\frac{\hbar^{2}}{2 m^{*}} \mathbf{k}^{2}
$$

where $\mathbf{k}$ is a wave vector on the Fermi surface. For the propagation of the particle along a trajectory on the Fermi surface,

TABLE II. Experimental data used for the AMRO calculations

\begin{tabular}{lcc}
\hline \hline Values & Electron band & Hole band \\
\hline$R^{*}[18]$ & 1.6 & 1.8 \\
$m^{*}[38]$ & 0.24 & 0.29 \\
$\tau[50]$ & $8.5 \mathrm{~cm}^{-1}$ & $8.5 \mathrm{~cm}^{-1}$ \\
$n[38]$ & $6.7 \times 10^{19} \mathrm{~cm}^{-3}$ & $6.1 \times 10^{19} \mathrm{~cm}^{-3}$ \\
$n[19]$ & $3.23 \times 10^{19} \mathrm{~cm}^{-3}$ & $3.05 \times 10^{19} \mathrm{~cm}^{-3}$ \\
$n_{\text {calc }}$ & $5 \times 10^{19} \mathrm{~cm}^{-3}$ & $5 \times 10^{19} \mathrm{~cm}^{-3}$ \\
\hline \hline
\end{tabular}

we use the following equation of motion:

$$
\hbar \frac{d \mathbf{k}}{\mathrm{d} t}=q \mathbf{v}(\mathbf{k}) \times \mathbf{B}(\theta, \varphi)
$$

where $\mathbf{B}$ is the applied magnetic field at specific angles $\theta$ and $\varphi$. The integral over time was taken from 0 to $9 \tau$, giving time for the particle to do more than one revolution around the Fermi surface and for the integral to converge. In the case of $\mathrm{EuB}_{6}$ the scattering rate $1 / \tau$ reported by Ref. [50] $1 / \tau=$ $8.5 \mathrm{~cm}^{-1}=4.05 \times 10^{10} \mathrm{~s}^{-1}$ is smaller than the cyclotron frequency $\left(\omega_{c}=\frac{e B(1 \mathrm{~T})}{2 \pi m^{*}}=1.2 \times 10^{11} \mathrm{~s}^{-1}\right)$. This means that in $\mathrm{EuB}_{6}$ the electrons complete more than one revolution around the Brillouin zone before they scatter. Also, in $\mathrm{EuB}_{6}$ the Fermi pockets are far from the Brillouin zone boundaries. As a consequence, for $\mathrm{EuB}_{6}$ the contribution from an anisotropic scattering rate can be neglected. This is very different from the situation in $\mathrm{Tl}_{2} \mathrm{Ba}_{2} \mathrm{Cu}_{1} \mathrm{O}_{6+\delta}$ [76]. In order to speed up calculations, we also assumed a uniform density of the charge carriers. All the values used the calculations are found in Table II.
[1] M. P. Lilly, K. B. Cooper, J. P. Eisenstein, L. N. Pfeiffer, and K. W. West, Phys. Rev. Lett. 82, 394 (1999).

[2] R. A. Borzi, S. A. Grigera, J. Farrell, R. S. Perry, S. J. S. Lister, S. L. Lee, D. A. Tennant, Y. Maeno, and A. P. Mackenzie, Science 315, 214 (2007).

[3] S. A. Kivelson, E. Fradkin, and V. J. Emery, Nature (London) 393, 550 (1998).

[4] A. H. MacDonald and M. P. A. Fisher, Phys. Rev. B 61, 5724 (2000).

[5] S.-W. Cheong, G. Aeppli, T. E. Mason, H. Mook, S. M. Hayden, P. C. Canfield, Z. Fisk, K. N. Clausen, and J. L. Martinez, Phys. Rev. Lett. 67, 1791 (1991).

[6] J. M. Tranquada, J. D. Axe, N. Ichikawa, A. R. Moodenbaugh, Y. Nakamura, and S. Uchida, Phys. Rev. Lett. 78, 338 (1997).

[7] Y. Ando, K. Segawa, S. Komiya, and A. N. Lavrov, Phys. Rev. Lett. 88, 137005 (2002).

[8] V. Hinkov, D. Haug, B. Fauque, P. Bourges, Y. Sidis, A. Ivanov, C. Bernhard, C. T. Lin, and B. Keimer, Science 319, 597 (2008).

[9] B. J. Ramshaw, N. Harrison, S. E. Sebastian, S. Ghannadzadeh, K. A. Modic, D. A. Bonn, W. N. Hardy, R. Liang, and P. A. Goddard, npj Quantum Mater. 2, 8 (2017).

[10] T.-M. Chuang, M. P. Allan, J. Lee, Y. Xie, N. Ni, S. L. Bud'ko, G. S. Boebinger, P. C. Canfield, and J. C. Davis, Science 327, 181 (2010).

[11] R. M. Fernandes, A. V. Chubukov, and J. Schmalian, Nat. Phys. 10, 97 (2014).
[12] M. D. Watson, T. K. Kim, A. A. Haghighirad, N. R. Davies, A. McCollam, A. Narayanan, S. F. Blake, Y. L. Chen, S. Ghannadzadeh, A. J. Schofield, M. Hoesch, C. Meingast, T. Wolf, and A. I. Coldea, Phys. Rev. B 91, 155106 (2015).

[13] I. I. Pomeranchuk, J. Exp. Theor. Phys. 8, 361 (1959).

[14] L. Dell'Anna and W. Metzner, Phys. Rev. Lett. 98, 136402 (2007).

[15] H. Yamase and W. Metzner, Phys. Rev. Lett. 108, 186405 (2012).

[16] F. Ronning, T. Helm, K. R. Shirer, M. D. Bachmann, L. Balicas, M. K. Chan, B. J. Ramshaw, R. D. McDonald, F. F. Balakirev, M. Jaime et al., Nature (London) 548, 313 (2017).

[17] S. V. Demishev, V. N. Krasnorussky, A. V. Bogach, V. V. Voronov, N. Y. Shitsevalova, V. B. Filipov, V. V. Glushkov, and N. E. Sluchanko, Sci. Rep. 7, 17430 (2017).

[18] M. C. Aronson, J. L. Sarrao, Z. Fisk, M. Whitton, and B. L. Brandt, Phys. Rev. B 59, 4720 (1999).

[19] X. Zhang, S. von Molnár, Z. Fisk, and P. Xiong, Phys. Rev. Lett. 100, 167001 (2008).

[20] S. Massidda, A. Continenza, T. M. de Pascale, and R. Monnier, Phys. B: Condens. Matter 102, 83 (1996).

[21] H. J. Tromp, P. van Gelderen, P. J. Kelly, G. Brocks, and P. A. Bobbert, Phys. Rev. Lett. 87, 016401 (2001).

[22] C. O. Rodriguez, R. Weht, and W. E. Pickett, Phys. Rev. Lett. 84, 3903 (2000). 
[23] J. Kim, W. Ku, C.-C. Lee, D. S. Ellis, B. K. Cho, A. H. Said, Y. Shvyd'ko, and Y.-J. Kim, Phys. Rev. B 87, 155104 (2013).

[24] S. Süllow, I. Prasad, M. C. Aronson, J. L. Sarrao, Z. Fisk, D. Hristova, A. H. Lacerda, M. F. Hundley, A. Vigliante, and D. Gibbs, Phys. Rev. B 57, 5860 (1998).

[25] L. Degiorgi, E. Felder, H. R. Ott, J. L. Sarrao, and Z. Fisk, Phys. Rev. Lett. 79, 5134 (1997).

[26] S. Süllow, I. Prasad, M. C. Aronson, S. Bogdanovich, J. L. Sarrao, and Z. Fisk, Phys. Rev. B 62, 11626 (2000).

[27] M. Pohlit, S. Rößler, Y. Ohno, H. Ohno, S. von Molnár, Z. Fisk, J. Müller, and S. Wirth, Phys. Rev. Lett. 120, 257201 (2018).

[28] P. Nyhus, S. Yoon, M. Kauffman, S. L. Cooper, Z. Fisk, and J. Sarrao, Phys. Rev. B 56, 2717 (1997).

[29] M. L. Brooks, T. Lancaster, S. J. Blundell, W. Hayes, F. L. Pratt, and Z. Fisk, Phys. Rev. B 70, 020401(R) (2004).

[30] X. Zhang, L. Yu, S. von Molnár, Z. Fisk, and P. Xiong, Phys. Rev. Lett. 103, 106602 (2009).

[31] P. Das, A. Amyan, J. Brandenburg, J. Müller, P. Xiong, S. von Molnár, and Z. Fisk, Phys. Rev. B 86, 184425 (2012).

[32] R. S. Manna, P. Das, M. de Souza, F. Schnelle, M. Lang, J. Müller, S. von Molnár, and Z. Fisk, Phys. Rev. Lett. 113, 067202 (2014).

[33] J. Kim, Y.-J. Kim, J. Kuneš, B. K. Cho, and E. J. Choi, Phys. Rev. B 78, 165120 (2008).

[34] L. G. L. Wegener and P. B. Littlewood, Phys. Rev. B 66, 224402 (2002)

[35] G. Beaudin, A. Désilets-Benoit, R. Arnold, S. Samothrakitis, K. Stenning, M. Nicklas, S. Gerber, N. Egetenmeyer, J. L. Gavilano, M. Kenzelmann, R. Cubitt, C. Dewhurst, M. Laver, and A. D. Bianchi (unpublished).

[36] W. Henggeler, H. Ott, D. P. Young, and Z. Fisk, Solid State Commun. 108, 929 (1998).

[37] B. J. Campbell, H. T. Stokes, D. E. Tanner, and D. M. Hatch, J. Appl. Crystallogr. 39, 607 (2006).

[38] G. A. Wigger, R. Monnier, H. R. Ott, D. P. Young, and Z. Fisk, Phys. Rev. B 69, 125118 (2004).

[39] K. Yamaji, J. Phys. Soc. Jpn 58, 1520 (1989).

[40] R. Yagi, Y. Iye, T. Osada, and S. Kagoshima, J. Phys. Soc. Jpn 59, 3069 (1990).

[41] C. Bergemann, A. P. Mackenzie, S. R. Julian, D. Forsythe, and E. Ohmichi, Adv. Phys. 52, 639 (2003).

[42] N. E. Hussey, M. Abdel-Jawad, A. Carrington, A. P. Mackenzie, and L. Balicas, Nature (London) 425, 814 (2003).

[43] R. R. Urbano, P. G. Pagliuso, C. Rettori, S. B. Oseroff, J. L. Sarrao, P. Schlottmann, and Z. Fisk, Phys. Rev. B 70, 140401(R) (2004).

[44] V. Glushkov, M. Anisimov, A. Bogach, S. Demishev, V. Ivanov, N. Samarin, A. Dukhnenko, N. Shitsevalova, K. Flachbart, and N. Sluchanko, J. Phys.: Conf. Ser. 150, 022014 (2009).

[45] Z. Fisk, D. C. Johnston, and B. Cornut, J. Appl. Phys. 50, 4 (1979).

[46] R. G. Goodrich, N. Harrison, J. J. Vuillemin, A. Teklu, D. W. Hall, Z. Fisk, D. Young, and J. Sarrao, Phys. Rev. B 58, 14896 (1998).

[47] M. Abdel-Jawad, M. P. Kennett, L. Balicas, A. Carrington, A. P. Mackenzie, R. H. McKenzie, and N. E. Hussey, Nat. Phys. 2, 821 (2006).

[48] J. D. Denlinger, J. A. Clack, J. W. Allen, G.-H. Gweon, D. M. Poirier, C. G. Olson, J. L. Sarrao, A. D. Bianchi, and Z. Fisk, Phys. Rev. Lett. 89, 157601 (2002).
[49] R. G. Chambers, Proc. Phys. Soc. Sect. A 65, 458 (1952).

[50] C. S. Snow, S. L. Cooper, D. P. Young, Z. Fisk, A. Comment, and J.-P. Ansermet, Phys. Rev. B 64, 174412 (2001).

[51] C. E. Matt, C. G. Fatuzzo, Y. Sassa, M. Månsson, S. Fatale, V. Bitetta, X. Shi, S. Pailhès, M. H. Berntsen, T. Kurosawa, M. Oda, N. Momono, O. J. Lipscombe, S. M. Hayden, J.-Q. Yan, J.-S. Zhou, J. B. Goodenough, S. Pyon, T. Takayama, H. Takagi et al., Phys. Rev. B 92, 134524 (2015).

[52] P. Kapitza, Proc. R. Soc. A 123, 292 (1929).

[53] H. Jones and C. Zener, Proc. R. Soc. A 145, 268 (1934).

[54] S.-Y. Gao, S. Xu, H. Li, C.-J. Yi, S.-M. Nie, Z.-C. Rao, H. Wang, Q.-X. Hu, X.-Z. Chen, W.-H. Fan, J.-R. Huang, Y.-B. Huang, N. Pryds, M. Shi, Z.-J. Wang, Y.-G. Shi, T.-L. Xia, T. Qian, and H. Ding, Phys. Rev. X 11, 021016 (2021)

[55] V. Barzykin and L. P. Gor'kov, Phys. Rev. Lett. 84, 2207 (2000).

[56] M. E. Zhitomirsky, T. M. Rice, and V. I. Anisimov, Nature (London) 402, 251 (1999).

[57] L. Balents and C. M. Varma, Phys. Rev. Lett. 84, 1264 (2000).

[58] D. P. Young, D. Hall, M. E. Torelli, Z. Fisk, J. L. Sarrao, J. D. Thompson, H.-R. Ott, S. B. Oseroff, R. G. Goodrich, and R. Zysler, Nature (London) 397, 412 (1999).

[59] T. Terashima, C. Terakura, Y. Umeda, N. Kimura, H. Aoki, and S. Kunii, J. Phys. Soc. Jpn. 69, 2423 (2000).

[60] K. Matsubayashi, M. Maki, T. Moriwaka, T. Tsuzuki, T. Nishioka, C. H. Lee, A. Yamamoto, T. Ohta, and N. K. Sato, J. Phys. Soc. Jpn. 72, 2097 (2003).

[61] M. Bałanda, Acta Phys. Pol. A 124, 964 (2013).

[62] A. Bianconi and N. Saini, Stripes and Related Phenomena, Selected Topics in Superconductivity (Springer US, New York, 2006).

[63] C. H. Booth, J. L. Sarrao, M. F. Hundley, A. L. Cornelius, G. H. Kwei, A. Bianchi, Z. Fisk, and J. M. Lawrence, Phys. Rev. B 63, 224302 (2001).

[64] D. D. Awschalom and M. E. Flatté, Nat. Phys. 3, 153 (2007).

[65] J. F. Rodríguez and J. A. Blanco, Phys. Scr. 71, CC19 (2005).

[66] B. Coqblin, The Electronic Structure of Rare-Earth Metals and Alloys: The Magnetic Heavy Rare-Earths (Academic Press, New York, 1977).

[67] B. Ambrosini, J. L. Gavilano, P. Vonlanthen, H. R. Ott, D. P. Young, and Z. Fisk, Phys. Rev. B 60, 3361 (1999).

[68] A. Aharoni, J. Appl. Phys. 83, 3432 (1998).

[69] J. M. D. Coey, Magnetism and Magnetic Materials (Cambridge University Press, Cambridge, 2001).

[70] S. Süllow, I. Prasad, S. Bogdanovich, M. C. Aronson, J. L. Sarrao, and Z. Fisk, J. Appl. Phys. 87, 5591 (2000).

[71] P. Majumdar and P. B. Littlewood, Nature (London) 395, 479 (1998).

[72] W. A. Phelan, S. M. Koohpayeh, P. Cottingham, J. A. Tutmaher, J. C. Leiner, M. D. Lumsden, C. M. Lavelle, X. P. Wang, C. Hoffmann, M. A. Siegler, N. Haldolaarachchige, D. P. Young, and T. M. McQueen, Sci. Rep. 6, 20860 (2016).

[73] S. M. Thomas, X. Ding, F. Ronning, V. Zapf, J. D. Thompson, Z. Fisk, J. Xia, and P. F. S. Rosa, Phys. Rev. Lett. 122, 166401 (2019).

[74] R. J. Balcombe, Proc. R. Soc. London 275, 113 (1963).

[75] O. Ciftja, Phys. Scr. 94, 105806 (2019).

[76] J. G. Analytis, M. Abdel-Jawad, L. Balicas, M. M. J. French, and N. E. Hussey, Phys. Rev. B 76, 104523 (2007). 